Case A $37+5$ weeks female infant born at Sligo University Hospital presented at the time of birth with a right upper extremity consistent with ischemia with evidence of gangrene. Following stabilisation of the infant in NICU, immediate transfer to a tertiary Paediatric Hospital was requested. There was uncertainty as to which speciality would accept the baby and multiple contacts with Neonatology, General Surgery and Plastic Surgery were necessary. There was significant difficulty procuring a bed and transport. The referring hospital transferred the baby at $51 / 2$ hours of life to a tertiary hospital where the baby was diverted to the Emergency department and subsequently admitted under shared neonatal and surgical care. US Doppler examination identified no pulses distal to the elbow joint with small non occlusive thrombus in IVC. The limb was conservatively managed for two weeks at which point below elbow amputation of the right upper limb was done. Cranial ultrasound and hypercoagulable workup were normal and no identifiable cause for the thrombus has been found. The baby remains well with full early intervention support.

Discussion Neonates with a clinically significant arterial thrombosis that develops in utero or during delivery are often born with or quickly develop gangrenous changes, thus illustrating the importance of prompt intervention. However, management guidelines for neonatal arterial thrombosis have not been well established. When an arterial thrombosis occurs perinatally, it is difficult to determine the duration of occlusion, and limb ischemia and loss may be imminent. The neonate in this case was born with gangrenous changes in the right arm due to an extensive thrombus and was subsequently managed with below elbow amputation with rehabilitation. The lack of a clear pathway of referral for neonates with thrombosis is evident in this case.

Conclusion A clear pathway of referral for neonates with arterial thrombosis should be established nationally and should include priority bed allocation and emergency transport.

\section{P68 WHEN A VIRUS HAS DIFFERENT FACES}

${ }^{1}$ Sofia Siena, ${ }^{1}$ Rosa Canestrale, 'Rossella Giorgio, ${ }^{2}$ Carmela De Meco, ${ }^{2}$ Irene Rutigliano, ${ }^{2}$ Pasquale Pio Maccarone, ${ }^{1}$ Francesca Lotti, ${ }^{1}$ Enrica Manca*, ${ }^{1}$ Agostino Petraccaro, ${ }^{1}$ Michaela Sciacovelli, ${ }^{1}$ Jessica Cinalski, ${ }^{1}$ Isabella Patisso, ${ }^{1}$ Annarita Pedico, ${ }^{1}$ Loredana Dipasquale, ${ }^{2}$ Michele Carmine Sacco, ${ }^{2}$ Massimo Pettoello Mantovani. ${ }^{1}$ Residency program in Pediatrics, University of Foggia, Foggia, Italy; ${ }^{2}$ Department of Pediatrics, Pediatric Unit, 'Casa Sollievo della Sofferenza' Scientific Institute, University of Foggia, San Giovanni Rotondo, Italy

\subsection{6/archdischild-2019-epa.423}

Measles is a highly contagious viral illness, characterized by fever, malaise, cough, coryza and conjunctivitis, followed by maculopapular exanthema, which spreads cephalocaudally and centrifugally. Measles, mumps, rubella (MMR) vaccination has led to the interruption of measles virus transmission and gives protection to unvaccinated individuals via herd immunity. The morbilliform exanthema can be found in various conditions, including infectious mononucleosis. It is characterized by fever, pharyngitis, lymphadenopathy and a generalized maculopapular, urtical or petechial rash occasionally can be present, especially after administration of beta-lactams. CASE REPORT: 17 months old male was admitted in our pediatric department for the appearance, 4 days earlier, of rash and fever $\left(\mathrm{T} 38,8^{\circ} \mathrm{C}\right)$. The exanthema consisted of an erythematous, maculopapular, blanching rash, which began on the face and progressed to the truck and extremities involving the palms and soles. In some areas it showed confluent and hemorrhagic features. The physical examination showed the presence of laterocervical lymphadenopathy, nonpurulent conjunctivitis and pharyngitis. About 10 days earlier it was administered antibiotic therapy with Amoxicillin for a fever associated with malaise, cough and coryza. The child had no history of allergies and the MMR vaccine was repeatedly delayed and eventually not carried out for multiple episodes of respiratory infections. The laboratory tests showed leucocitosis with a normal differential count, mild elevation of transaminases, elevation of inflammatory markers and $\mathrm{LDH}$; the morphological evaluation of the peripheral smear showed some activated lymphomonocitoid cells. Given the rash characteristics and the strong suspicion of measles, the patient was located in isolation and infectivological tests were performed (TORCH, Monotest, Respiratory Multiplex PCR panel and a serology for measles). They all came back negative except for the anti VCA IgM for EBV infection. The patient was treated with IV fluids and antipyretics. Antibiotic therapy was administered in order to prevent bacterial superinfections. After 72 hours the rash started to darken and then to gradually fade. The patient was dismissed with the diagnosis of maculopapular exanthema in mononucleosis infection. Clinical manifestations of infectious mononucleosis can be similar to those of measles and, especially in unvaccinated patients, can sometimes be confused with it. Maculopapular exanthema can be found in various conditions, such as common viral or bacterial infections, IgA vasculitis, Kawasaki disease or drug eruption. For this reason, it is important to consider mononucleosis in the differential diagnosis of measles, especially in case of hemorrhagic and infiltrated rash, not much described in the literature.

\section{P69 TAKAYASU ARTERITIS PRESENTING IN AN 11-YEAR-OLD BOY}

${ }^{1}$ Ana Louise Hawke*, ${ }^{2}$ Danielle O'Connor, ${ }^{2}$ Emma Jane MacDermott, ${ }^{2}$ Orla G Killeen. ' Our Lady's Children's Hospital, Crumlin, Dublin, Ireland; ${ }^{2}$ Our Lady's Children's Hospital, Dublin, Ireland

\subsection{6/archdischild-2019-epa.424}

Introduction We present a case of Takayasu arteritis in a Caucasian Irish 11 year old male.

Background Takayasu Arteritis, which usually involves the Aorta and it's main branches is an idiopathic granulomatous vasculitis of unknown aetiology with significant associated morbidity and mortality.

It is rare in children, usually presents in Asian adult females and has a worldwide incidence of 2.6/1000,000.

Case Presentation A previously well Caucasian eleven year old boy, with no past medical history of note, presented with an insidious onset of persistent, intermittent abdominal, flank and back pain, wakening him form sleep and exacerbated by exercise.

Histologically confirmed appendicitis with appendicectomy was made 4 weeks prior to presentation to his local hospital.

Examination was normal, including pulses and 4 limb Blood Pressure measurements. Inflammatory markers on presentation- CRP(90) and ESR(120)- were markedly raised. Full blood count, renal and liver profiles were normal. 